\title{
NON-LINEARITIES IN A QUASI-LINEAR SYSTEM SUBJECTED TO EXTERNAL AND PARAMETRIC EXCITATIONS OF DIFFERENT ORDERS
}

\author{
NGUYEN VAN DINH \\ Institute of Mechanics, NCNST
}

In [1], using the multiple scale method, A. N. Nayfeh has studied the interaction of fundamental parametric resonance with subharmonic resonance. Essentially, the system considered belongs to. the class of oscillating ones, subjected simultaneously to two excitations (external and parametric). of different orders of smallness ( $\varepsilon$ and $\varepsilon^{2}$, respectively). It has been shown that, depending on the dephase between two excitations the system may be enhanced or extinguished.

Below, to obtain some more informations about this phenomenon, we shall plot the resonance curve of the combined oscillation. The asymptotic method [2] will be used, the roles of quadratic and cubic non-linearities will be analysed.

\section{§1. SYSTEM UNDER CONSIDERATION AND ITS AVERAGED EQUATION}

Let us consider an oscillating system described by the differential equation:

$$
\ddot{x}+\omega^{2} x=\varepsilon\left\{\beta x^{2}+q \cos 2 \omega t\right\}+\varepsilon^{2}\left\{-h \dot{x}+\Delta x-\gamma x^{3}+2 p x \cos 2(\omega t+\sigma)\right\}
$$

where: $x$ - an oscillatory variable; $\varepsilon>0$ - a small parameter; $\beta, \gamma$ - coefficients of quadratic and cubic non-linearities, respectively; $q>0,2 p>0$ and $2 \omega, \sigma(0 \leq \sigma<\pi)$ - intensities, common frequency and dephase between the external and parametric excitations, respectively, $\Delta=\left(\omega^{2}-1\right)$ - the detuning parameter ( 1 - the natural frequency). Using the asymptotic method, in the second approximation, the solution of (1.1) will be found in the form:

$$
\begin{aligned}
& x=a \cos \psi+\varepsilon u_{1}(a, \theta, \psi)+\varepsilon^{2} u_{2}(a, \theta, \psi) \\
& \dot{a}=\varepsilon A_{1}(a, \theta)+\varepsilon^{2} A_{2}(a, \theta) \\
& \dot{\theta}=\varepsilon B_{1}(a, \theta)+\varepsilon^{2} B_{2}(a, \theta), \quad \psi=\omega t+\theta
\end{aligned}
$$

where: $a, \theta$ - slowly varying amplitude and dephase of the oscillatory regime; $u_{1}, u_{2}\left(A_{1}, B_{1}, A_{2}\right.$, $B_{2}$ ) - unknown functions of $a, \theta, \varphi(a, \theta)$ which are periodic relative to $\theta, \psi(\theta)$ with period $2 \pi$.

Introducing (1.2) into (1.1), using (1.3) comparing the terms of like powers of $\varepsilon$ then, those of same harmonics of $\psi$, we obtain:

$$
\begin{gathered}
A_{1}=B_{1}=0 \\
u_{1}=\frac{1}{\omega^{2}}\left\{\frac{\beta a^{2}}{2}-\frac{\beta a^{2}+2 q \cos 2 \theta}{6} \cos 2 \psi-\frac{q \sin 2 \theta}{3} \sin 2 \psi\right\} \\
\dot{a}=-\frac{\varepsilon^{2}}{2 \omega} a\left\{h \omega+\left(g \cos 2 \sigma-\frac{\beta q}{3 \omega^{2}}\right) \sin 2 \theta-(p \sin 2 \sigma) \cos 2 \theta\right\} \\
a \dot{\theta}=-\frac{\varepsilon^{2}}{2 \omega} a\left\{\left[\Delta-\left(\frac{3 \gamma}{4}-\frac{5 \beta^{2}}{6 \omega^{2}}\right) a^{2}\right]+(p \sin 2 \sigma) \sin 2 \theta+\left(p \cos 2 \sigma-\frac{\beta q}{3 \omega^{2}}\right) \cos 2 \theta\right\}
\end{gathered}
$$




\section{§2. PURE FORCED OSCILLATION}

The equations (1.5) admit the trivial solution $a=0$, corresponding to the pure forced oscillation:

$$
x=\varepsilon u_{1}=-\frac{\varepsilon q}{3 \omega^{2}}\{\cos 2 \psi \cos 2 \theta+\sin 2 \psi \sin 2 \theta\}=-\frac{\varepsilon}{3 \omega^{2}} \cos 2 \omega t
$$

The stability study of this "quasi equilibrium regime" can be based on the following variational system [3]:

$$
\begin{aligned}
& \dot{a}=-\frac{\varepsilon^{2} a}{2 \omega}\left\{h \omega+\left(p \cos 2 \sigma-\frac{\beta q}{3 \omega^{2}}\right) \sin 2 \theta-(p \sin 2 \sigma) \cos 2 \theta\right\} \\
& 0=-\frac{\varepsilon^{2}}{2 \omega}\left\{\Delta+(p \sin 2 \sigma) \sin 2 \theta+\left(p \cos 2 \sigma-\frac{\beta q}{3 \omega^{2}}\right) \cos 2 \theta\right\}
\end{aligned}
$$

Let us write (2.2b) as:

$$
-\Delta=(p \sin 2 \sigma) \sin 2 \theta+\left(p \cos 2 \sigma-\frac{\beta q}{3 \omega^{2}}\right) \cos 2 \theta
$$

and, by $X$, we denote:

$$
X=\left(p \cos 2 \sigma-\frac{\beta q}{3 \omega^{2}}\right) \sin 2 \theta-(p \sin 2 \sigma) \cos 2 \theta
$$

From (2.3a), (2.3b), it is easy to deduce:

$$
X= \pm \sqrt{\left(p \cos 2 \sigma-\frac{\beta q}{3 \omega^{2}}\right)^{2}+(p \sin 2 \sigma)^{2}-\Delta^{2}}
$$

The stability conditions are of the form:

$$
\operatorname{Re}\left\{h \omega \pm \sqrt{\left(p \cos 2 \sigma-\frac{\beta q}{3 \omega^{2}}\right)^{2}+(p \sin 2 \sigma)^{2}-\Delta^{2}}\right\}>0
$$

Since $h>0, \omega \approx 1$, only the second inequality (sign - ) is retained:

$$
h \omega>\sqrt{\left(p \cos 2 \sigma-\frac{\beta q}{3 \omega^{2}}\right)^{2}+(p \sin 2 \sigma)^{2}-\Delta^{2}}
$$

or

$$
\Delta^{2}>\left(p \cos 2 \sigma-\frac{\beta q}{3 \omega^{2}}\right)^{2}+(p \sin 2 \sigma)^{2}-h^{2} \omega^{2}
$$

\section{§3. COMBINED OSCILLATION}

More interesting stationary regime is the combined oscillation of amplitude $a_{0}$ and dephase $\theta_{0}$, determined from the equation:

$$
\begin{aligned}
& \left(p \cos 2 \sigma-\frac{\beta p}{3 \omega^{2}}\right) \sin 2 \theta-(p \sin 2 \sigma) \cos 2 \theta=-h \omega \\
& (p \sin 2 \sigma) \sin 2 \theta+\left(p \cos 2 \sigma-\frac{\beta q}{3 \omega^{2}}\right) \cos 2 \theta=\left[\left(\frac{3 \gamma}{4}-\frac{5 \beta^{2}}{6 \omega^{2}}\right) a^{2}-\Delta\right]
\end{aligned}
$$

After eliminating $\theta$, we obtain the resonance curve

$$
W=\left[\left(\frac{3 \gamma}{4}-\frac{5 \beta^{2}}{6 \omega^{2}}\right) a^{2}-\Delta\right]^{2}+h^{2} \omega^{2}-\left(p \cos 2 \sigma-\frac{\beta q}{3 \omega^{2}}\right)^{2}-p^{2} \sin ^{2} 2 \sigma=0
$$


To study the stability of this regime, we introduce the variations $\delta a=a-a_{0}, \delta \theta=\theta-\theta_{0}$ in, establish the variational equations:

$$
\begin{aligned}
& \delta \dot{a}=-\frac{\varepsilon^{2} a}{\omega}\left\{\left(\frac{3 \gamma}{4}-\frac{5 \beta^{2}}{6 \omega^{2}}\right) a^{2}-\Delta\right\} \delta \theta \\
& \delta \dot{\theta}=\frac{\varepsilon^{2}}{\omega a}\left(\frac{3 \gamma}{4}-\frac{5 \beta^{2}}{6 \omega^{2}}\right) a^{2} \delta a-\varepsilon^{2} h \delta \theta
\end{aligned}
$$

The characteristic equation of (3.3) is:

$$
\rho^{2}+\varepsilon^{2} h \rho+\frac{\varepsilon^{4}}{\omega^{2}}\left\{\left(\frac{3 \gamma}{4}-\frac{5 \beta^{2}}{6 \omega^{2}}\right) a^{2}-\Delta\right\}\left(\frac{3 \gamma}{4}-\frac{5 \beta^{2}}{6 \omega^{2}}\right) a^{2}=0
$$

Since $h>0$, the stability condition is given by the inequality:

$$
\left[\left(\frac{3 \gamma}{4}-\frac{5 \beta^{2}}{6 \omega^{2}}\right) a^{2}-\Delta\right]\left(\frac{3 \gamma}{4}-\frac{5 \beta^{2}}{6 \omega^{2}}\right)>0
$$

, in compact form:

$$
\frac{\partial W}{\partial a^{2}}>0
$$

Remark. As it has been shown in [4], we must examine also the critical case where the terminant (of the algebraic equation (3.1))

$$
D=\left|\begin{array}{cc}
\left.p \cos 2 \sigma-\frac{\beta q}{3 \omega^{2}}\right) & -p \sin 2 \sigma \\
p \sin 2 \sigma & \left(p \cos 2 \sigma-\frac{\beta q}{3 \omega^{2}}\right)
\end{array}\right|=\left(p \cos 2 \sigma-\frac{\beta q}{3 \omega^{2}}\right)^{2}+p^{2} \sin ^{2} 2 \sigma=0
$$

However, form (3.7), we deduce $\sigma=0, \pi / 2, \omega^{2}=\beta q / 3 p$ so that the algebraic equations (3.1) Imit no solution

\section{§4. ROLE OF QUADRATIC AND CUBIC NON-LINEARITIES}

The relationship (3.2) can be written as:

$$
\left(\frac{3 \gamma}{4}-\frac{5 \beta^{3}}{6 \omega^{2}}\right) a^{2}=\Delta \pm \sqrt{\left(p \cos 2 \sigma-\frac{\beta q}{3 \omega^{2}}\right)^{2}+(p \sin 2 \sigma)^{2}-h^{2} \omega^{2}}
$$

For simplicity, we suppose that $\beta>0, \gamma>0$. Obviously, the resonance curve may exists only

$$
Z=\left(\beta \cos 2 \sigma-\frac{\beta q}{3 \omega^{2}}\right)^{2}+p^{2} \sin ^{2} 2 \sigma-h^{2} \omega^{2} \geq 0
$$

For illustration, let us fix $h^{2}=0.00006 ; \beta=0.1 ; p=0.012 ; q=0.12$. In figure 1 , (a) epresents the straight line $z_{2}=h^{2} \omega^{2}$ and (b) (c) (d) (e) (f) (g) represent the curves $Z_{1}=$ $\left(p \cos 2 \sigma-\frac{\beta q}{3 \omega^{2}}\right)^{2}+p^{2} \sin ^{2} 2 \sigma$ corresponding to $\sigma=0, \pi / 12, \pi / 6, \pi / 3, \pi / 2,2 \pi / 3$ respectively if $r=0$, the equation $z=0$ admits three solutions $\omega_{1}^{2}<\omega_{2}^{2}<\omega_{3}^{2}$ and the resonance curve may :xists when $z \geq 0$ i.e. if $\omega^{2} \leq \omega_{1}^{2}$ or $\omega_{2}^{2} \leq \omega^{2} \leq \omega_{3}^{2}$. For each other chosen values $\sigma$, we have only ine solution $\bar{\omega}$ and $z \geq 0$ if $\omega^{2} \leq \bar{\omega}^{2}$.

However, the existence of the resonance curve depends also and notably on the "ratio" between ihe quadratic non-linearity and the cubic one, more exactly, on the value $\omega_{*}^{2}=\frac{5 \beta^{2}}{6} / \frac{3 \gamma}{4}$, vanishing the coefficient $k\left(\omega^{2}\right)=\frac{3 \gamma}{4}-\frac{5 \beta^{2}}{6 \omega^{2}}$. 


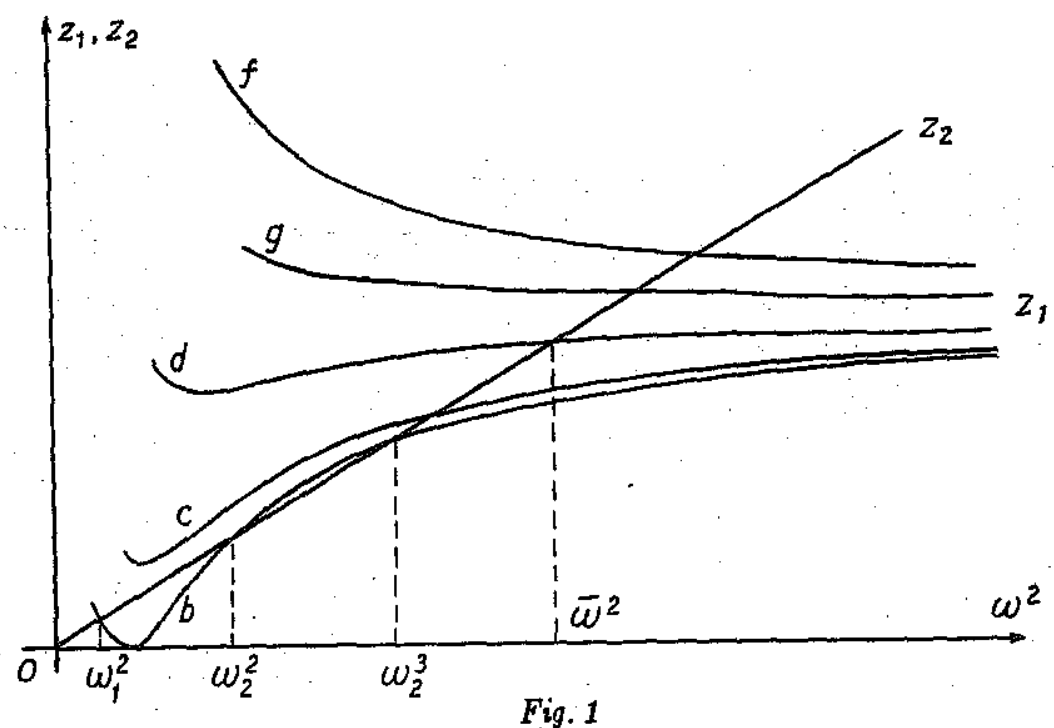

Let us denote the right-hand side of $(4.1)$ by:

$$
y=\omega^{2}-1 \pm \sqrt{\left(p \cos 2 \sigma-\frac{\beta q}{3 \omega^{2}}\right)^{2}+(p \sin 2 \sigma)^{2}-h^{2} \omega^{2}}
$$

Assume that the graphic (a) of $y$, exists in the interval $I\left(\omega_{1}^{2} \leq \omega^{2} \leq \omega_{2}^{2}\right)$ and is of the shape given in figure 2.

- If $\omega_{*}^{2}<\omega_{1}^{2}$, then $k\left(\omega^{2}\right)>0$ in the interval $I$, therefore the resonance curve $a^{2}=$ $y / k\left(\omega^{2}\right)$ is given by the curve of shape $\left(b_{1}\right)$

- If $\omega_{*}^{2}>\omega_{2}^{2}$, then $k\left(\omega^{2}\right)<0$ in the interval $I$, therefore the resonance curve $a^{2}$ is given by the curve of shape $\left(b_{2}\right)$

- If $\omega_{1}^{2}<\omega_{*}^{2}<\omega_{2}^{2}$, the resonance curve consists of two branches $\left(b_{3}\right)$ and $\left(b_{4}\right)$, admitting as asymptote the ordinate line of abscissa $\omega^{2}=\omega_{*}^{2}$

In reality, depending on the chosen order of smallness, the acceptable parts of the resonance curve are nearly straight lines.

Some typical resonance curve are given in figures $3,4$.

It is clearly that the soft-hardness of the oscillating system depends on $\omega_{*}^{2}$. If $\omega_{*}^{2}$ is small enough, the resonance curve leans to the right, the system belongs to hard type; on the contrary, if $\omega_{*}^{2}$ is large enough, the resonance curve leans to the left, the system belongs to soft-type; if $\omega_{*}^{2}$ is in the neighbourhood of 1 , the system is neutralized, it becomes a linear one.

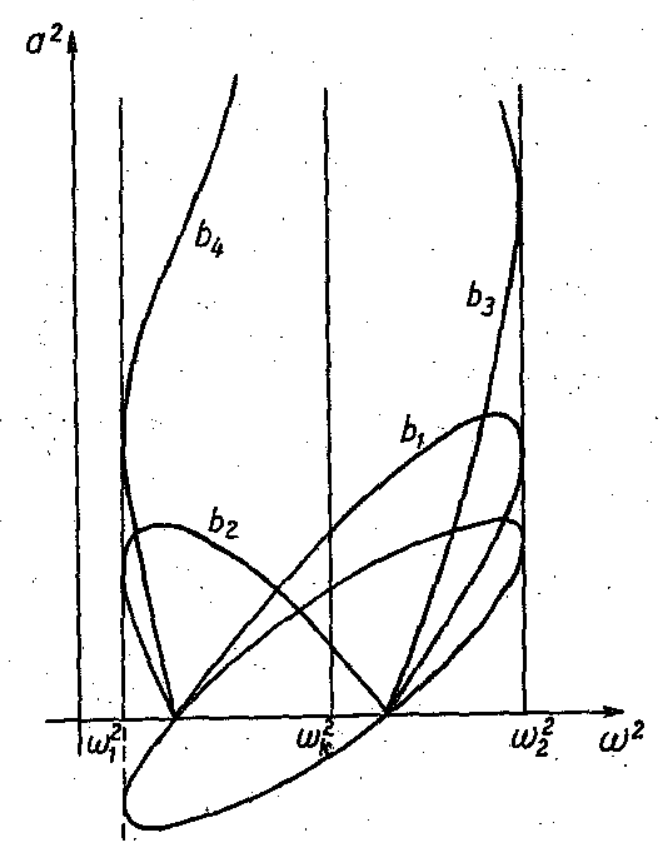

Fig. 2 


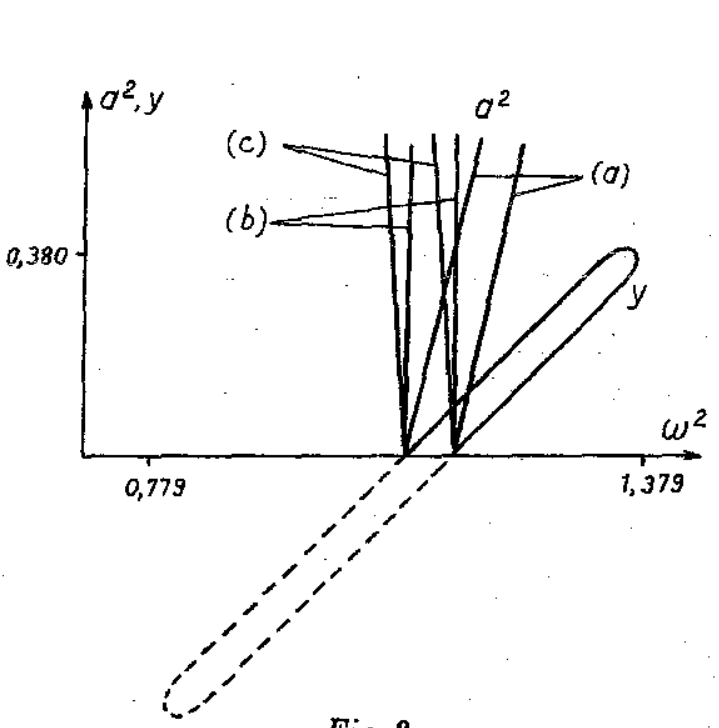

Fig.s

$$
\begin{gathered}
h^{2}=0.0006, p=0.012, q=0,12, \\
\beta=0.1, \sigma=0
\end{gathered}
$$

(a): $\gamma=0.005$ (b): $\gamma=0.04$ (c): $\gamma=0.01$

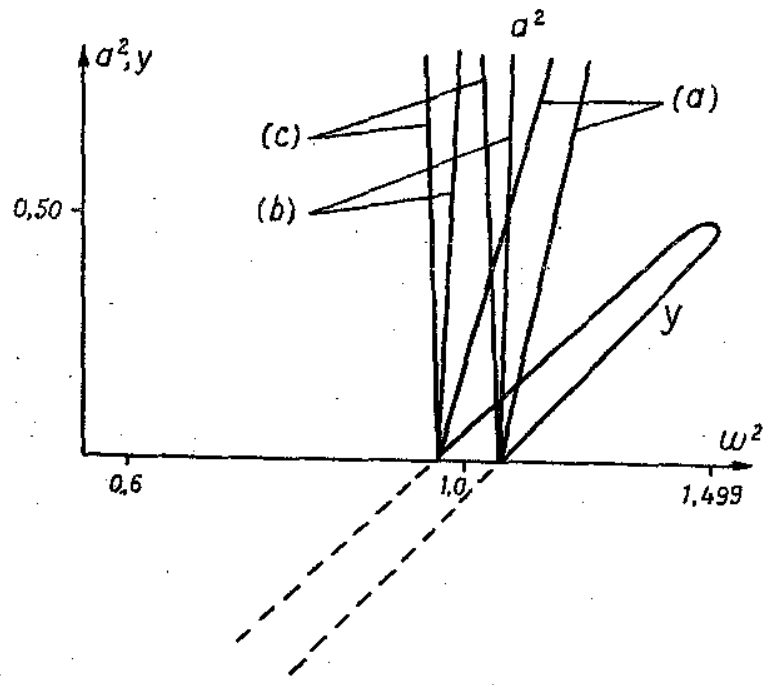

Fig. 4

$h^{2}=0.0001, p=0.012, q=0,12$,

$$
\beta=0.1, \sigma=0.785
$$

(a): $\gamma=0.005$ (b): $\gamma=0.04$ (c): $\gamma=0.01$

\section{CONCLUSION}

We have examined a quasi-linear oscillating system subjected simultaneously to external excitation of order $\varepsilon$ in subharmonic resonance and parametric excitation of order $\varepsilon^{2}$ in fundamental resonance. It has been found if the quadratic and cubic non-linearities are of order $\varepsilon$ and $\varepsilon^{2}$, respectively, the soft-hardness of the system depends on both these non-linearities; in particular case, the system examined can be neutralised.

This publication is completed with financial support from the National Basic Research program in National Sciences.

\section{REFERENCE}

1. Nayfeh A. H. Interaction of fundamental parametric resonances with subharmonic resonance of order one-half J. Sound and Vibration 96(3), 1984, 330-340.

2. Bogoliubov N. N., Mitropolskii Yu. A. Asymptotic methods in the theory of non-linear oscillation, Moscow, 1963.

3. Nguyen Van Dinh. On the dephase angle in a variational system of the equilibrium regime. J. of Mechanics, NCNST of Vietnam, No 1, 1994, 29-35.

Received December 4, 1994

\section{PHI TUYẾN TRONG HÊ Á TUYẾN CHỊU KÍCH ĐộNG NGOÀI VÀ THÔNG SÓ Ở CẤP KHÁC NHAU}

Đã khảo sát hệ dao động á tuyến chịu kích động ngoài cấp $\varepsilon$ cộng hướng $1 / 2$ và kích động thông số cấp $\varepsilon^{2}$ cộng hướng chính. Nhận thấy rằng nếu các yếu tố phi tuyến đàn hồi bậc hai và bậc ba tương ứng $̛$ cấp $\varepsilon$ và $\varepsilon^{2}$ thì tính cứng - mềm của hệ sẽ phụ thuộc vào cả hai yểu tố đó; trường hợp riêng, hệ có thể bị trung hòa. 